\title{
A study on the relationship between morphological lesions of lupus nephritis with demographic and biochemical findings
}

\author{
Nasrin Tavassoli ${ }^{\circledR}$, Hamid Nasri ${ }^{1^{*}}{ }^{(\mathbb{D}}$, Rohollah Valizadeh $^{{ }^{\circledR}}$ \\ 'Department of Internal Medicine, Isfahan University of Medical Sciences, Isfahan, Iran \\ ${ }^{2}$ Minimally Invasive Surgery Research Center, Hazrat-e Rasool General Hospital, Iran University of Medical Sciences, Tehran, \\ Iran and Student Research Committee, Department of Epidemiology, School of Public Health, Iran University of Medical \\ Sciences, Tehran, Iran
}

Correspondence to:

Prof. Hamid Nasri,

Email: hamidnasri@yahoo.com

hamidnasri@med.mui.ac.ir

Received: 29 June 202 Accepted: 10 Oct. 2021 ePublished: 31 Oct. 2021

Keywords: Lupus nephritis, Systemic lupus erythematosus, Proteinuria

\begin{abstract}
Introduction: Lupus nephritis is one of the important aspects of systemic lupus erythematosus (SLE). Objectives: This study aimed to investigate possible relationship between pathological lesions of lupus nephritis classes and demographic and biochemical findings among patients.

Patients and Methods: This is a cross-sectional study that was conducted on a group of renal biopsy proven lupus nephritis patients using lupus nephritis classification of ISN/RPS 2003. We collected demographic data of all patients including age, gender serum creatinine and $24 \mathrm{~h}$ proteinuria.

Results: Data of 101 patients, of whom 78 (77.23\%) were females and mean age of $33.54 \pm 13.15$ years. The mean serum creatinine and proteinuria were $1.54 \pm 0.88 \mathrm{mg} / \mathrm{dL} 2502.5 \pm 1495.05 \mathrm{mg} / \mathrm{d}$. Based on our data, IV-G (class IV, diffuse lupus nephritis-global) lupus nephritis class was the most common (39.6\%) followed by class III (23.8\%). The mean crescent and sclerotic glomeruli were $1.66 \pm 3.32$ and $2.27 \pm 5.32$, respectively. In this study, 24 hours proteinuria, serum creatinine, activity percent, chronicity percent, crescent and glomerular sclerosis between genders showed no significant differences $(P>0.05)$. The correlation between plasma creatinine and activity was directly positive and significant $(r=0.381, P=0.001)$. In addition, a significant correlation between $\mathrm{C} 1 \mathrm{q}$ deposits and glomerular sclerosis $(P=0.031)$ was detected. Accordingly, a significant correlation between IgG deposits and lupus nephritis classification $(P=0.025)$ was seen.

Conclusion: Lupus nephritis of IV-G and III classes of lupus nephritis were most common among patients and higher IgG deposits were observed in patients with IV-G classification. We found a significant correlation between glomerular sclerosis and $\mathrm{C} 1 \mathrm{q}$ deposits that could be an indicator of lupus nephritis activity and severity. However, we recommend further studies in this regard.
\end{abstract}

Introduction

Renal involvement is one of the most serious manifestations of systemic lupus erythematosus (SLE) and is associated with increased mortality and disability (1). Studies that have assessed SLE have reported that the patients' 4-5-year survival rate in 1950 was $50 \%$, which has recently increased to $80 \% 15$-year survival (2-4). While there have been no developments in renal failure issues in SLE patients and so far there is no explanation for this apparent discrepancy in this issue $(5,6)$.

The most common findings in patients with lupus nephritis include proteinuria, urinary casts, hematuria, pyuria, foamy urine, low serum albumin, ankle edema, elevated serum creatinine, and hypertension (7). Kidney biopsy is essential to determine the type of involvement, as it significantly affects disease management. Most patients with lupus show a series of abnormal

\begin{abstract}
Key point
In a study on 101 lupus nephritis patients, with mean age of $33.54 \pm 13.15$ years, we found IV-G (class IV diffuse lupus nephritis-global) lupus nephritis class was the most common $(39.6 \%)$ followed by class III (23.8\%).
\end{abstract}

findings when examining a biopsy specimen with a light microscope or more specialized techniques such as immunofluorescence (IF) or electron microscopy (8). Apart from glomerulonephritis, kidney involvement in lupus can be caused by interstitial nephritis, tubular disease, thrombotic microangiopathy, vasculitis, arteriosclerosis, and lupus vasculopathy $(9,10)$.

Classification of lupus nephritis is based on changes seen in light microscopy and IF staining (11-13) as follows:

Class I; Minimal mesangial lupus nephritis; accumulation of immune complex deposits in

Copyright (C) 2022 The Author(s); Published by Society of Diabetic Nephropathy Prevention. This is an open-access article distributed under the terms of the Creative Commons Attribution License (http://creativecommons.org/licenses/by/4.0), which permits unrestricted use, distribution, and reproduction in any medium, provided the original work is properly cited. 
the mesangium. Glomeruli in this class are normal under a light microscope, but mesenchymal deposits are visible on IF. Class II; mesangial proliferative lupus nephritis; with light microscopy, a clear increase in mesangial cellularity or expansion of the mesangial matrix can be seen. Mesangial immune deposits are observed which is detectable by electron microscopy or IF, which is not visible with light microscopy. Class III: focal lupus nephritis; It is seen as active or inactive focal glomerulonephritis, segmental or global glomerular involvement in less than $50 \%$ of all glomeruli. Class IV; diffuse lupus nephritis, which is usually a proliferative glomerulonephritis, segmental or global, which affects $50 \%$ or more of all glomeruli. It is typically characterized by diffuse sub-endothelial immune deposits. Class V; membranous lupus nephritis. Class VI; advanced lupus nephritis sclerosis; in this case 90\% and more of the glomerulus is involved. In this class, global glomerular sclerosis is seen with or without residual activity. This stage is the end-stage of all previous classes.

Factors that are generally accepted as factors associated with the prognosis and high risk of developing renal failure in lupus nephritis include; demographic, laboratory and histologic findings. Previous studies have indicated that pathological lesions of lupus nephritis classes have correlations with demographic and biochemical findings of patients but few studies have been conducted in Iranian populations.

\section{Objectives}

Regarding the importance and prevalence of lupus nephritis and considering the possible correlations between lupus nephritis classes and demographic variables, we aimed to investigate a study on the possible relationship of demographic and biochemical with morphologic variables in lupus nephritis patients undergoing renal biopsy.

\section{Patients and Methods}

\section{Study design}

This is a cross-sectional study that was performed in 20082020 in Khorshid and Al-Zahra hospitals affiliated to Isfahan University of Medical Sciences. The current study was conducted on medical documents of patients that were diagnosed with lupus nephritis.

The total number of our patients was 101 whose SLE was confirmed based on SLICC (Systemic Lupus International Collaborating Clinics) SLE criteria and renal biopsy with IF staining since lupus nephritis classes were determined based on ISN/RPS 2003.

Patients with other medical diseases that could influence on kidneys (including co-existence of diabetes, IgA nephropathy or other glomerulopathies such as amyloidosis) did not enter the study. These patients were recognized based on patient's medical documents and physician's note. Furthermore, patients with incomplete demographic or laboratory data and biopsy reports did not enter the study.
During the study duration, data of 101 eligible patients were studied using census method. We collected demographic data of all patients including age and gender. Data regarding serum creatinine and $24 \mathrm{~h}$ proteinuria were collected from laboratory documents of patients.

We should mention that laboratory biopsy included two samples; one sample for IF microscope and one sample for light microscope. Levels of IgA, IgG, IgM, C3 and C1q antibodies were measured by IF microscopy. IF findings were scored based on their intensity of deposits (0 to $3+$ ). The definitive diagnosis of lupus nephritis was approved by abundant granular deposits of $\mathrm{C} 1 \mathrm{q}, \mathrm{IgG}$ and $\mathrm{C} 3$, which is called "full-house". By the light microscopy, morphological lesions including proliferation in mesangial, endocapillary and extra-capillary and also global and segmental and percentage of lupus nephritis activity in the glomeruli were examined. Based on these, the lupus class was determined.

\section{Data analysis}

Data of demographic, laboratory and pathologic were collected and analyzed in order to assess possible correlations. The obtained data were entered into the Statistical Package for Social Sciences (SPSS) version 24. Quantitative data were reported as mean \pm standard deviation and qualitative data as frequency distribution (percentage). We used student $t$ test for data analysis. Pearson's correlation was used as well. $P$ value $<0.05$ was considered as significance threshold.

\section{Results}

In the present study, medical documents of 101 patients were enrolled. The mean age of the patients was $33.54 \pm$ 13.15 years (range 11 to 79 years). The study population consisted of 78 females (77.23\%) and 23 males (22.77\%). The mean serum creatinine and proteinuria were $1.54 \pm$ $0.88 \mathrm{mg} / \mathrm{dL}$ and $2502.5 \pm 1495.05 \mathrm{mg} /$ day respectively. The mean crescent and sclerotic glomeruli were $1.66 \pm$ 3.32 and $2.27 \pm 5.32$, respectively. In this study, IV-G lupus nephritis class was the most common (39.6\%) followed by class III (23.8\%), since the classes V, IV-S and VI were $17.8 \%, 2 \%$ and $1 \%$ of the total classes, respectively. Around $11.9 \%$ of lupus nephritis classes were mixed classes, including V+II (3\%), V+IV-S (1\%), V+IV-G (1\%) and $\mathrm{V}+\mathrm{III}(6.9 \%)$. The most common mixed class was V+III (6.9\%) (Table 1). We evaluated data of IgG, IgM, $\mathrm{C} 3$ and $\mathrm{C1q}$ immunofluorescence findings and their possible relationship with age, gender, lupus nephritis classification, $24 \mathrm{~h}$ proteinuria, serum creatinine, crescents and glomerular sclerosis. Evaluation of IgG, IgM, C3 and $\mathrm{Clq}$ antibodies showed no significant relationships between antibody deposit intensity and demographic and laboratory parameters and also crescents $(P>0.05$; Tables 2-5). This study showed a significant relationship between IgG deposits and lupus nephritis classification $(P=0.025)$ with higher IgG deposits were observed in patients with IV-G lupus nephritis class. Our data also 
Table 1. Frequency of different lupus nephritis classifications

\begin{tabular}{llcc}
\hline Variable & & No. & Percentage of total \\
\hline \multirow{6}{*}{ Class } & I & 1 & 1 \\
& II & 3 & 3 \\
& III & 24 & 23.8 \\
& IV-S (S=segmental) & 2 & 2 \\
& IV-G (G=global) & 40 & 39.6 \\
& V & 18 & 17.8 \\
& VI & 1 & 1 \\
& Total & 89 & 88.1 \\
\hline \multirow{4}{*}{ Mixed class } & V+II & 3 & 3 \\
& V+IV-S & 1 & 1 \\
& V+IV-G & 1 & 1 \\
& V+III & 7 & 6.9 \\
& Total & 12 & 11.9 \\
\hline \multirow{6}{*}{} & & &
\end{tabular}

indicated a significant relationship between $\mathrm{C} 1 \mathrm{q}$ intensity and number of sclerotic glomeruli because the number of glomerular scleroses were significantly higher in intensity of 2 and more for $\mathrm{Clq}$ antibody ( $P=0.031$; Table 5$)$. The mean serum creatinine in females and males was $1.5 \pm 0.8$ $\mathrm{mg} / \mathrm{dL}$ and $1.7 \pm 1 \mathrm{mg} / \mathrm{dL}$ respectively $(P>0.05)$. Moreover, the mean $24 \mathrm{~h}$ proteinuria of both genders was $2572 \pm 1546$ $\mathrm{mg} /$ day and $2267 \pm 1311 \mathrm{mg} / \mathrm{d}$ respectively $(P>0.05)$. The mean activity percent was $49 \pm 32 \%$ in females and $54 \pm 34 \%$ in males and the mean chronicity percent was $15 \pm 17 \%$ in females and $9 \pm 13 \%$ in males. The mean crescent in females and males were $1.64 \pm 3.45$ and $1.74 \pm 2.93$ and the mean sclerotic glomeruli in females and males were $2.3 \pm 5.65$ and $2.3 \pm 4.2$, respectively. Accordingly, quantity of proteinuria, serum creatinine levels, disease activity, disease chronicity, crescent and glomerular sclerosis between genders showed no significant differences among genders $(P>0.05$; Table $6)$. The correlation between plasma creatinine and activity was directly positive and significant $(\mathrm{r}=0.381, P=0.001)$, the correlation between plasma creatinine and chronicity was not significant $(P=0.065, P=0.578$; Table 7 , Figures 1 and 2).

\section{Discussion}

Evaluation of demographic and laboratory findings and their correlations to lupus nephritis could have high clinical and preventive importance. In the present study, we investigated data of 101 cases with lupus nephritis with IF study. Based on our data, the classifications of IV-G and III were most common among patients. We also observed that higher IgG deposits was observed in patients with IV-G lupus nephritis class. We also observed that the number of sclerotic glomeruli were significantly higher in intensity of 2 and more for C1q. These data could be indicative of higher lupus nephritis activity with higher intensity of IgG antibody.

So far, some studies have investigated the possible relationships between lupus nephritis classes and demographic variables.

In 2021, Nie and colleagues investigated the characteristics of patients with lupus nephritis and the pathological report of them. They reported that IV-G was the most common lupus nephritis classification among patients with lupus nephritis (14). Tao et al also evaluated

Table 2. Relationships between IgG grade and the study variables

\begin{tabular}{|c|c|c|c|c|c|c|}
\hline Variable & & Grade 0 & Grade 1 & Grade 2 & Grade 3 & $P$ value \\
\hline \multirow{2}{*}{ Gender, No. (\%) } & Female & $4(100.0)$ & $2(66.7)$ & $22(91.7)$ & $50(71.4)$ & \multirow{2}{*}{0.110} \\
\hline & Male & $0(0.0)$ & $1(33.3)$ & $2(8.3)$ & $20(28.6)$ & \\
\hline \multirow{11}{*}{ Class, No. (\%) } & I & $1(25.0)$ & $0(0.0)$ & $0(0.0)$ & $0(0.0)$ & \multirow{11}{*}{0.025} \\
\hline & II & $0(0.0)$ & $0(0.0)$ & $2(8.3)$ & $1(1.4)$ & \\
\hline & III & $1(25.0)$ & $0(0.0)$ & $3(12.5)$ & $20(28.6)$ & \\
\hline & IV-S & $0(0.0)$ & $1(33.3)$ & $0(0.0)$ & $1(1.4)$ & \\
\hline & IV-G & $1(25.0)$ & $1(33.3)$ & $11(45.8)$ & $27(38.6)$ & \\
\hline & V & $1(25.0)$ & $0(0.0)$ & $4(16.7)$ & $13(18.6)$ & \\
\hline & VI & $0(0.0)$ & $1(33.3)$ & $0(0.0)$ & $0(0.0)$ & \\
\hline & $V+I I$ & $0(0.0)$ & $0(0.0)$ & $2(8.3)$ & $1(1.4)$ & \\
\hline & $V+I V-S$ & $0(0.0)$ & $0(0.0)$ & $0(0.0)$ & $1(1.4)$ & \\
\hline & $V+I V-G$ & $0(0.0)$ & $0(0.0)$ & $1(4.2)$ & $0(0.0)$ & \\
\hline & $V+I I I$ & $0(0.0)$ & $0(0.0)$ & $1(4.2)$ & $6(8.6)$ & \\
\hline \multicolumn{2}{|l|}{ Age $($ mean $\pm \mathrm{SD})$} & $27 \pm 7$ & $31 \pm 16$ & $35 \pm 11$ & $34 \pm 14$ & 0.769 \\
\hline \multicolumn{2}{|c|}{ 24-h Proteinuria (mean \pm SD) } & $925 \pm 869$ & $2500 \pm 500$ & $2482 \pm 1370$ & $2600 \pm 1555$ & 0.228 \\
\hline \multicolumn{2}{|c|}{ Serum creatinine (mean $\pm S D$ ) } & $1.3 \pm 0.6$ & $1.9 \pm 0.7$ & $1.6 \pm 0.8$ & $1.5 \pm 0.9$ & 0.761 \\
\hline \multicolumn{2}{|c|}{ Crescent $($ mean $\pm \mathrm{SD})$} & $3 \pm 6$ & $3 \pm 4$ & $2 \pm 5$ & $1 \pm 2$ & 0.854 \\
\hline \multicolumn{2}{|c|}{ Sclerotic glomeruli $($ mean \pm SD) } & $1 \pm 1$ & $9 \pm 10$ & $2 \pm 3$ & $2 \pm 6$ & 0.089 \\
\hline
\end{tabular}


Table 3. Relationships between IgM grade and the study variables

\begin{tabular}{|c|c|c|c|c|c|}
\hline Variable & & Score 0 & Score 1 & Score 2 & $P$ value \\
\hline \multirow{2}{*}{ Gender, N (\%) } & Female & $28(80.0)$ & 30 (78.9) & $20(71.4)$ & \multirow{2}{*}{0.687} \\
\hline & Male & $7(20.0)$ & 8 (21.1) & 8 (28.6) & \\
\hline \multirow{11}{*}{ Class, N (\%) } & I & $1(2.9)$ & $0(0.0)$ & $0(0.0)$ & \multirow{11}{*}{0.743} \\
\hline & II & $1(2.9)$ & $2(5.3)$ & $0(0.0)$ & \\
\hline & III & $11(31.4)$ & $7(18.4)$ & $6(21.4)$ & \\
\hline & IV-S & $0(0.0)$ & $2(5.3)$ & $0(0.0)$ & \\
\hline & IV-G & $11(31.4)$ & $14(36.8)$ & 15 (53.6) & \\
\hline & V & $5(14.3)$ & 7 (18.4) & $6(21.4)$ & \\
\hline & $\mathrm{VI}$ & $1(2.9)$ & $0(0.0)$ & $0(0.0)$ & \\
\hline & $V+I I$ & $1(2.9)$ & $2(5.3)$ & $0(0.0)$ & \\
\hline & $V+I V-S$ & $0(0.0)$ & $1(2.6)$ & $0(0.0)$ & \\
\hline & V+IV-G & $1(2.9)$ & $0(0.0)$ & $0(0.0)$ & \\
\hline & $V+I I I$ & $3(8.6)$ & $3(7.9)$ & $1(3.6)$ & \\
\hline \multicolumn{2}{|l|}{ Age $($ mean \pm SD) } & $32 \pm 13$ & $34 \pm 12$ & $35 \pm 15$ & 0.356 \\
\hline \multicolumn{2}{|c|}{ 24-h Proteinuria $($ mean \pm SD) } & $2308 \pm 1429$ & $2400 \pm 1017$ & $2885 \pm 2019$ & 0.115 \\
\hline \multicolumn{2}{|c|}{ Serum creatinine (mean \pm SD) } & $1.5 \pm 0.8$ & $1.5 \pm 0.9$ & $1.6 \pm 1.0$ & 0.762 \\
\hline \multicolumn{2}{|c|}{ Crescent $($ mean $\pm \mathrm{SD})$} & $2 \pm 3$ & $2 \pm 4$ & $2 \pm 3$ & 0.875 \\
\hline \multicolumn{2}{|c|}{ Sclerotic glomeruli (mean \pm SD) } & $2 \pm 4$ & $3 \pm 7$ & $2 \pm 4$ & 0.918 \\
\hline
\end{tabular}

Table 4. Relationships between C3 grade score and the study variables

\begin{tabular}{|c|c|c|c|c|c|c|}
\hline Variable & & Score 0 & Score 1 & Score 2 & Score 3 & $P$ value \\
\hline \multirow{2}{*}{ Gender, N (\%) } & Female & $1(100.0)$ & $16(84.2)$ & $19(65.5)$ & $42(80.8)$ & \multirow{2}{*}{0.362} \\
\hline & Male & $0(0.0)$ & $3(15.8)$ & $10(34.5)$ & $10(19.2)$ & \\
\hline \multirow{11}{*}{ Class, N (\%) } & 1 & $0(0.0)$ & $1(5.3)$ & $0(0.0)$ & $0(0.0)$ & \multirow{11}{*}{0.134} \\
\hline & II & $0(0.0)$ & $1(5.3)$ & $1(3.4)$ & $1(1.9)$ & \\
\hline & III & $0(0.0)$ & $5(26.3)$ & $11(37.9)$ & $8(15.4)$ & \\
\hline & IV-S & $0(0.0)$ & $1(5.3)$ & $0(0.0)$ & $1(1.9)$ & \\
\hline & IV-G & $0(0.0)$ & $5(26.3)$ & $11(37.9)$ & $24(46.2)$ & \\
\hline & V & $1(100.0)$ & $2(10.5)$ & $4(13.8)$ & $11(21.2)$ & \\
\hline & $\mathrm{VI}$ & $0(0.0)$ & $1(5.3)$ & $0(0.0)$ & $0(0.0)$ & \\
\hline & $V+I I$ & $0(0.0)$ & $2(10.5)$ & $0(0.0)$ & $1(1.9)$ & \\
\hline & $V+I V-S$ & $0(0.0)$ & $0(0.0)$ & $0(0.0)$ & $1(1.9)$ & \\
\hline & $V+I V-G$ & $0(0.0)$ & $0(0.0)$ & $1(3.4)$ & $0(0.0)$ & \\
\hline & $V+I I I$ & $0(0.0)$ & $1(5.3)$ & $1(3.4)$ & $5(9.6)$ & \\
\hline \multicolumn{2}{|c|}{ Age (mean $\pm \mathrm{SD})$} & 31 & $37 \pm 14$ & $33 \pm 14$ & $32 \pm 12$ & 0.246 \\
\hline \multicolumn{2}{|c|}{ 24-h Proteinuria (mean \pm SD) } & 296 & $1989 \pm 1060$ & $2579 \pm 1367$ & $2690 \pm 1651$ & 0.081 \\
\hline \multicolumn{2}{|c|}{ Serum creatinine (mean $\pm \mathrm{SD}$ ) } & 0.8 & $1.4 \pm 0.5$ & $1.7 \pm 0.8$ & $1.5 \pm 1.0$ & 0.861 \\
\hline \multicolumn{2}{|c|}{ Crescent $($ mean $\pm \mathrm{SD})$} & 0 & $1 \pm 3$ & $2 \pm 3$ & $2 \pm 4$ & 0.352 \\
\hline \multicolumn{2}{|c|}{ Sclerotic glomeruli $($ mean \pm SD) } & 1 & $5 \pm 10$ & $2 \pm 4$ & $1 \pm 2$ & 0.246 \\
\hline
\end{tabular}

C3 or C1q deposition in kidneys in 2020. It was indicated that $\mathrm{C} 1 \mathrm{q}$ deposits were significantly correlated with lupus nephritis classification (mostly observed in IV-G) and was correlated with higher disease chronicity (15). In another study, Yung and colleagues showed no significant relationship between antibody deposits in renal tissue with demographic variables such as age, gender and serum creatinine concentration (16). The findings of our study were in line with the results of these reports. We showed that IV-G and III were most common among patients and higher IgG deposits were observed in patients with IV-G lupus nephritis classification.
In a previous study, Chen and colleagues reported that IV-G classification was most common in patients with lupus nephritis and mentioned a possible correlation between IgG deposits and lupus nephritis classification (17). These data were also consistent with the findings of our study.

We also found, the number of sclerotic glomeruli were significantly higher in intensity of 2 and more of C1q. We believe that this issue is due to the relationship between lupus severity and $\mathrm{Clq}(18,19)$, which implies further kidney damage. Recently, Shang et al evaluated C1q and its relationship to the lupus activity. They showed this 
Table 5. Relationships between C1q grade and the study variables

\begin{tabular}{|c|c|c|c|c|c|c|}
\hline Variable & & Score 0 & Score 1 & Score 2 & Score 3 & $P$ value \\
\hline \multirow{2}{*}{ Gender, N (\%) } & Female & $2(66.7)$ & $21(77.8)$ & $28(80.0)$ & $27(75.0)$ & \multirow{2}{*}{0.921} \\
\hline & Male & $1(33.3)$ & $6(22.2)$ & $7(20.0)$ & $9(25.0)$ & \\
\hline \multirow{11}{*}{ Class, N (\%) } & 1 & $1(33.3)$ & $0(0.0)$ & $0(0.0)$ & $0(0.0)$ & \multirow{11}{*}{0.104} \\
\hline & II & $0(0.0)$ & $2(7.4)$ & $1(2.9)$ & $0(0.0)$ & \\
\hline & III & $0(0.0)$ & $5(18.5)$ & $10(28.6)$ & $9(25.0)$ & \\
\hline & IV-S & $0(0.0)$ & $1(3.7)$ & $0(0.0)$ & $1(2.8)$ & \\
\hline & IV-G & $0(0.0)$ & 7 (25.9) & $15(42.9)$ & $18(50.0)$ & \\
\hline & V & $1(33.3)$ & $6(22.2)$ & $6(17.1)$ & $5(13.9)$ & \\
\hline & VI & $0(0.0)$ & $1(3.7)$ & $0(0.0)$ & $0(0.0)$ & \\
\hline & $\mathrm{V}+\mathrm{II}$ & $1(33.3)$ & $1(3.7)$ & $1(2.9)$ & $0(0.0)$ & \\
\hline & $V+I V-S$ & $0(0.0)$ & $0(0.0)$ & $0(0.0)$ & $1(2.8)$ & \\
\hline & $V+I V-G$ & $0(0.0)$ & $1(3.7)$ & $0(0.0)$ & $0(0.0)$ & \\
\hline & $\mathrm{V}+\mathrm{III}$ & $0(0.0)$ & $3(11.1)$ & $2(5.7)$ & $2(5.6)$ & \\
\hline \multicolumn{2}{|c|}{ Age (mean $\pm \mathrm{SD}$ ) } & $25 \pm 7$ & $36 \pm 12$ & $34 \pm 11$ & $32 \pm 16$ & 0.473 \\
\hline \multicolumn{2}{|c|}{ 24-h Proteinuria $($ mean $\pm \mathrm{SD})$} & $1508 \pm 2174$ & $2349 \pm 1347$ & $2579 \pm 1641$ & $2626 \pm 1424$ & 0.313 \\
\hline \multicolumn{2}{|c|}{ Serum creatinine (mean \pm SD) } & $1 \pm 0.3$ & $1.6 \pm 0.9$ & $1.5 \pm 0.7$ & $1.6 \pm 1.0$ & 0.509 \\
\hline \multicolumn{2}{|c|}{ Crescent $($ mean $\pm \mathrm{SD})$} & 0 & $2 \pm 3$ & $2 \pm 3$ & $2 \pm 4$ & 0.341 \\
\hline \multicolumn{2}{|c|}{ Sclerotic glomeruli $($ mean $\pm \mathrm{SD})$} & 0 & $2 \pm 4$ & $3 \pm 8$ & $1 \pm 2$ & 0.031 \\
\hline
\end{tabular}

biomarker could be an effective indicator of active lupus nephritis which could also possibly predict the kidney damage (20). Our data are suggestive of a relationship between lupus nephritis severity and activity with renal damage due to significantly higher rates of glomerular sclerosis.

In another recent study, Koopman et al investigated renal deposits of complement factors and their relationships to various factors in 183 patients. Based on their results, C1q and C3 deposits had no significant relationships with lupus nephritis classification, comorbidities and demographic factors or disease course (21).

We found a significant correlation between plasma creatinine and activity. In older studies, this relationship has been noted (22).

\section{Conclusion}

Taken together, we observed that IV-G and III classification of lupus nephritis were most common among patients and higher IgG deposits were observed in patients with IV-G lupus nephritis class. We also found significant correlation

Table 6. Assessments of 24-h proteinuria, serum creatinine, disease activity and chronicity by gender

\begin{tabular}{lccc}
\hline Variable & Female $($ mean \pm SD) & Male $($ mean \pm SD) & $\boldsymbol{P}$ value \\
\hline $\begin{array}{l}\text { 24h Proteinuria } \\
(\mathrm{mg} / \mathrm{d})\end{array}$ & $2572 \pm 1546$ & $2267 \pm 1311$ & 0.246 \\
$\begin{array}{l}\text { Serum creatinine } \\
(\mathrm{mg} / \mathrm{dL})\end{array}$ & $1.5 \pm 0.8$ & $1.7 \pm 1$ & 0.522 \\
Activity $(\%)$ & $49 \pm 32$ & $54 \pm 34$ & 0.692 \\
\hline Chronicity $(\%)$ & $15 \pm 17$ & $9 \pm 13$ & 0.270 \\
Crescent & $1.64 \pm 3.45$ & $1.74 \pm 2.93$ & 0.791 \\
\hline Sclerotic glomeruli & $2.3 \pm 5.65$ & $2.3 \pm 4.2$ & 0.532 \\
\hline
\end{tabular}

between glomerular sclerosis and $\mathrm{C} 1 \mathrm{q}$ deposits that could be an indicator of lupus nephritis activity. However, further studies are required in this regard.

\section{Limitations of the study}

The limitation of the current study was that we had a limited study population. Selected patients in this study are different from our previous study on lupus nephritis (22). Since in this study we selected the renal biopsies which all, had two fragments for IF and light microscopies. This investigation was also conducted in a single laboratory and requires further investigation by larger studies.

\section{Authors' contribution}

$\mathrm{HN}$ contributed to the design of the study and had leadership responsibility for the research. NT conducted the study and wrote the primary draft. RV conducted the statistical analysis. HN finalized the paper. All authors read and signed the final manuscript.

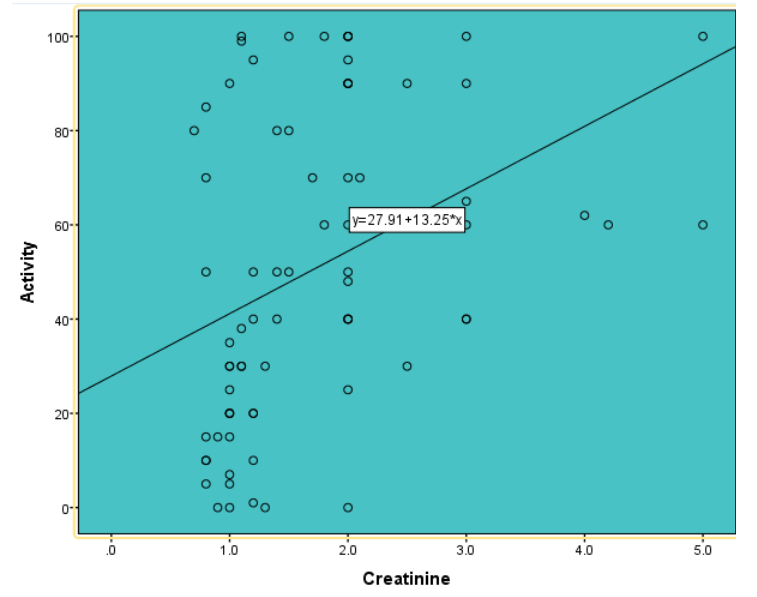

Figure 1. Correlation between serum creatinine and glomerular activity percent. 


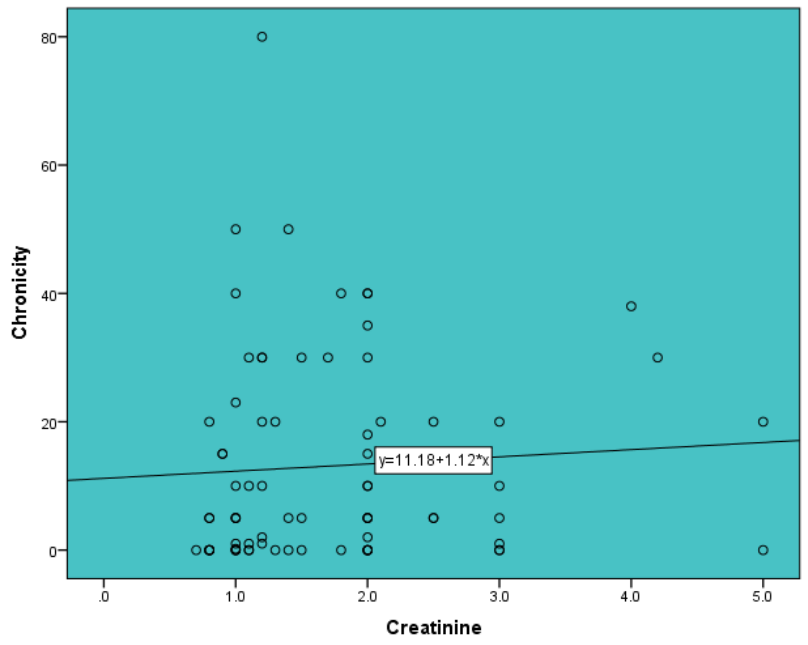

Figure 2. Correlation between serum creatinine and glomerular chronicity percent.

Table 7. Correlation among creatinine, activity and chronicity

\begin{tabular}{llcc}
\hline Pearson's correlation & Chronicity & Activity \\
\hline \multirow{2}{*}{ Creatinine } & Coefficient & 0.065 & 0.381 \\
& $P$ value & 0.578 & 0.001 \\
\hline
\end{tabular}

Conflicts of interest

The authors declare that they have no competing interests.

\section{Ethical issues}

The research followed the tenets of the Declaration of Helsinki. The Ethics Committee of Isfahan University of Medical Sciences approved this study. (IR.MUI.MED.REC.1399.1075). Accordingly, written informed consent was taken from all participants before any intervention. This study was extracted from M.D thesis of Nasrin Tavassoli at this university (Thesis \# 399973). Besides, ethical issues (including plagiarism, data fabrication, double publication) have been completely observed by the authors.

\section{Funding/Support}

This study was granted by Isfahan University of Medical Sciences (Grant \#48980).

\section{References}

1. Gustafsson JT, Herlitz Lindberg M, Gunnarsson I, Pettersson $\mathrm{S}$, Elvin K, et al. Excess atherosclerosis in systemic lupus erythematosus, -A matter of renal involvement: Case control study of 281 SLE patients and 281 individually matched population controls. PLoS One. 2017;12:e174572. doi: 10.1371/journal.pone.0174572.

2. Stojan G, Petri M. Epidemiology of systemic lupus erythematosus: an update. Curr Opin Rheumatol. 2018;30:144150. doi: 10.1097/BOR.0000000000000480.

3. Mejia-Vilet JM, Rovin BH. Epidemiology and Management of Lupus Nephritis. Dubois' Lupus Erythematosus and Related Syndromes. Elsevier; 2019. p. 727-44.

4. Gergianaki I, Bortoluzzi A, Bertsias G. Update on the epidemiology, risk factors, and disease outcomes of systemic lupus erythematosus. Best Pract Res Clin Rheumatol. 2018;32:188-205. doi: 10.1016/j.berh.2018.09.004.

5. Dall'Era M, Bruce IN, Gordon C, Manzi S, McCaffrey J, Lipsky PE. Current challenges in the development of new treatments for lupus. Ann Rheum Dis. 2019;78:729-735. doi: 10.1136/ annrheumdis-2018-214530.
6. Park DJ, Kang JH, Lee JW, Lee KE, Kim TJ, Park YW, et al. Risk factors to predict the development of chronic kidney disease in patients with lupus nephritis. Lupus. 2017;26:1139-1148. doi: 10.1177/0961203317694257.

7. Anders HJ, Saxena R, Zhao MH, Parodis I, Salmon JE, Mohan C. Lupus nephritis. Nat Rev Dis Primers. 2020;6:7. doi: 10.1038/ s41572-019-0141-9.

8. Moroni G, Depetri F, Ponticelli C. Lupus nephritis: When and how often to biopsy and what does it mean? J Autoimmun. 2016;74:27-40. doi: 10.1016/j.jaut.2016.06.006.

9. Barrera-Vargas A, Rosado-Canto R, Merayo-Chalico J, Arreola-Guerra JM, Mejía-Vilet JM, Correa-Rotter R, et al. Renal thrombotic microangiopathy in proliferative lupus nephritis: risk factors and clinical outcomes: a case-control study. J Clin Rheumatol. 2016;22:235-40. doi: 10.1097/ RHU.0000000000000425.

10. Kaul A, Agrawal V, Bhaduaria D, Agrawal V, Prasad N, Gupta A, et al. Vasculitis and vasculopathy in Lupus Nephritis: Clinical variability, outcome, and new insight into treatment. Saudi J Kidney Dis Transpl. 2017;28:415-24. doi: 10.4103/13192442.202777.

11. Stokes MB, D'Agati VD. Classification of Lupus Nephritis; Time for a Change? Adv Chronic Kidney Dis. 2019;26:323-9. doi: 10.1053/j.ackd.2019.06.002.

12. Dasari S, Chakraborty A, Truong L, Mohan C. A Systematic Review of Interpathologist Agreement in Histologic Classification of Lupus Nephritis. Kidney Int Rep. 2019;4:14201425. doi: 10.1016/j.ekir.2019.06.011.

13. Imran TF, Yick F, Verma S, Estiverne C, Ogbonnaya-Odor C, Thiruvarudsothy S, Reddi AS, Kothari N. Lupus nephritis: an update. Clin Exp Nephrol. 2016;20:1-13. doi: 10.1007/ s10157-015-1179-y.

14. Nie P, Hu L, Li B, Lou Y, Luo M, Wang Y, et al. Relationship between hyperuricemia and serositis in patients with lupus nephritis. Int Urol Nephrol. 2021 May 10. doi: 10.1007/ s11255-021-02873-z.

15. Tao J, Song D, Liu XL, Yu F, Zhao MH. Circulating anti-C3b IgG in lupus nephritis: A large cohort study. Clin Immunol. 2020;217:108514. doi: 10.1016/j.clim.2020.108514.

16. Yung S, Yap DY, Chan TM. Recent advances in the understanding of renal inflammation and fibrosis in lupus nephritis. F1000Res. 2017;6:874. doi: 10.12688/f1000research.10445.1.

17. Chen D, Hu W. Lupus podocytopathy: a distinct entity of lupus nephritis. J Nephrol. 2018;31:629-34. doi: 10.1007/s40620017-0463-1.

18. Ekinci Z, Ozturk K. Systemic lupus erythematosus with C1q deficiency: treatment with fresh frozen plasma. Lupus. 2018;27:134-138. doi: 10.1177/0961203317741565.

19. Emad G, Al-Barshomy SM. Anti-C1q antibodies in lupus nephritis and their correlation with the disease activity. Saudi J Kidney Dis Transpl. 2020;31:342-352. doi: 10.4103/13192442.284008.

20. Shang X, Ren L, Sun G, Yu T, Yao Y, Wang L, Liu F, Zhang L, He $X$, Liu M. Anti-dsDNA, anti-nucleosome, anti-C1q, and antihistone antibodies as markers of active lupus nephritis and systemic lupus erythematosus disease activity. Immun Inflamm Dis. 2021;9:407-18. doi: 10.1002/iid3.401.

21. Koopman JJE, Rennke HG, Leatherwood C, Speyer CB, D'Silva K, McMahon GM, et al. Renal deposits of complement factors as predictors of end-stage renal disease and death in patients with lupus nephritis. Rheumatology (Oxford). 2020;59(12):37513758. doi: 10.1093/rheumatology/keaa174.

22. Nasri H, Ahmadi A, Baradaran A, Momeni A, Nasri P, Mardani $S$, et al. Clinicopathological correlations in lupus nephritis; a single center experience. J Nephropathol. 2014;3:115-20. doi: 10.12860/jnp.2014.22. 